\title{
ON THE VALUES OF THE EPSTEIN ZETA FUNCTION
}

\author{
by JOHN RODERICK SMART $\dagger$
}

(Received 15 January, 1971; revised 16 August, 1971)

1. Introduction. Let $\zeta(s)=\Sigma n^{-s}(\operatorname{Re} s>1)$ denote the Riemann zeta function; then, as is well known, $\zeta(2 m)=2^{2 m-1} \pi^{2 m}\left|B_{2 m}\right| /(2 m)$ !, where $B_{m}$ denotes the $m$ th Bernoulli number, In this paper we investigate the possibility of similar evaluations of the Epstein zeta function $\zeta_{Q}(s)$ at the rational integers $s=k \geqq 2$. Let

$$
Q(m, n)=a m^{2}+2 b m n+c n^{2} \quad\left(a>0, a c-b^{2}=1\right)
$$

be a positive definite quadratic form and

$$
\zeta_{Q}(s)=\sum_{m, n}^{\prime} Q(m, n)^{-s} \quad(s=\sigma+i t, \operatorname{Re} s>1),
$$

where the summation is over all pairs of integers except $(0,0)$. In attempting to evaluate $\zeta_{Q}(k)$ we are guided by Kronecker's first limit formula [11]

$$
\lim _{s \rightarrow 1}\left[\zeta_{Q}(s)-\pi /(s-1)\right]=2 \pi\left(\gamma-\log 2-\log \sqrt{y}|\eta(\tau)|^{2}\right),
$$

where $\gamma$ is Euler's constant,

$$
\eta(\tau)=e^{2 \pi i \tau / 24} \prod_{m=1}^{\infty}\left(1-e^{2 \pi i m r}\right) \quad(\operatorname{Im} \tau>0)
$$

is the Dedekind eta-function, and $\tau$ is the complex number in the upper half plane, $\mathscr{H}$, associated with $Q$ by the formula

$$
Q(m, n)=y^{-1}|m+n \tau|^{2}, \quad \tau=x+i y=b / a+i / a .
$$

On the basis of (1.3) we would expect a formula involving functions of $\tau$. This formula is stated in Theorem 1, (2.13).

The Dedekind eta-function is a modular form of dimension $-\frac{1}{2}$ with a multiplier system [7]. It satisfies the functional equations

$$
\eta(\tau+1)=e^{2 \pi i / 24} \eta(\tau), \quad \eta(-1 / \tau)=e^{-2 \pi / 8} \sqrt{\tau} \eta(\tau) .
$$

The (inhomogeneous) modular group $\Gamma(1)$ consists of all linear fractional transformations $w=V \tau=(a \tau+b) /(c \tau+d)$ with rational integers $a, b, c$ and $d$ and $a d-b c=1$. It is known to be generated by

$$
w=U \tau=\tau+1 \text { and } w=T \tau=-1 / \tau .
$$

The homogeneous modular group $S L(2, \mathbf{Z})$ ( $\mathbf{Z}$ the integers) will be denoted by $\Gamma(1)$. The Eisenstein series

$$
G_{2 k}(\tau)=\sum_{m, n}^{\prime}(m+n \tau)^{-2 k} \quad(k=2,3,4, \ldots)
$$

† The research for this paper was partially supported by National Science Foundation grant GP12081. 
are modular forms of dimension $-2 k$ and multiplier system 1 [4]. Moreover

$$
G_{2 k}(\tau)=2 \zeta(2 k)+\frac{2(2 \pi i)^{2 k}}{\Gamma(2 k)} \sum_{n=1}^{\infty} \sigma_{2 k-1}(n) e(n \tau),
$$

where $\sigma_{2 k-1}(n)$ denotes the sum of the $2 k-1$ powers of the divisors of $n$, and we are using the notation

$$
e(z)=e^{2 \pi i z}
$$

In a certain sense $\log \eta(\tau)=\pi i \tau / 12-\sum_{1}^{\infty} \sigma_{-1}(n) e(n \tau)$ is an Eisenstein series of dimension 0 .

We find in evaluating $\zeta_{Q}(k)$ that in place of $\log \eta(\tau)$ in (1.3) we obtain in (2.13) functions $\varphi_{2 k}(\tau)$ and their derivatives, which are closely related to indefinite integrals of Eisenstein series. The functions $\varphi_{2 k}(\tau)$ satisfy the transformation formulas

and

$$
\varphi_{2 k}(\tau+1)=\varphi_{2 k}(\tau)+2 \zeta(2 k) / \tau(\tau+1)
$$

$$
\tau^{2 k-2} \varphi_{2 k}(-1 / \tau)=\varphi_{2 k}(\tau)+4 \sum_{j=1}^{k-1} \zeta(2 k-2 j) \zeta(2 j) \tau^{2 k-2 j-1} .
$$

We shall call them modular forms of dimension $2 k-2$ with rational period functions.

We are not able to evaluate $\zeta_{Q}(k)$ for any $Q$ or $k$ in the same sense as we know $\zeta(2 m)$. However, with the aid of (1.10) we are able to give explicit evaluations of some related series associated with two particular quadratic forms(those associated with $\tau=i$ and $\tau=\frac{1}{2}(-1+i \sqrt{ } 3$ )). Examples of such series are given in (4.8), (4.11) and (4.12).

Finally we indicate some applications to the problem of evaluating the Riemann zeta function for odd arguments. There is little more here than the rederivation of the results of Grosswald [4].

2. The value of $\zeta_{Q}(k)$. In order to obtain the connection between $\zeta_{Q}(k)$ and modular forms with rational period functions, we start with a formula of Selberg and Chowla [3], first proved by Rankin [9], in the form given by Bateman and Grosswald [2]:

$$
\zeta_{Q}(s)=2 y^{s} \zeta(2 s)+2 y^{1-s_{\zeta}}(2 s-1) \Gamma\left(s-\frac{1}{2}\right) \Gamma\left(\frac{1}{2}\right) / \Gamma(s)+2 \pi \sqrt[3]{y} H(s) / \Gamma(s),
$$

where

$$
H(s)=4 \sum_{n=1}^{\infty} n^{s-\frac{1}{2}} \sigma_{1-2 s}(n) \cos 2 \pi n x \times K_{s-\frac{1}{2}}(2 \pi y n) .
$$

In stating the formula of Bateman and Grosswald we have changed their notation to conform to (1.1) and (1.5). I would like to thank Bruce Berndt for suggesting the use of (2.1) in place of my earlier proof, which used the Poisson summation formula and the evaluation of the resulting integrals by means of the residue method. Now we set $s=k$, a positive integer, in (2.1) and (2.2) and we use the formula [13, p. 80 (12)]

$$
K_{k-\frac{1}{1}}(z)=\left(\frac{\pi}{2 z}\right)^{\frac{1}{2}} e^{-z} \sum_{r=0}^{k-1}(k-1+r) ! /\left\{r !(k-1-r) !(2 z)^{r}\right\}
$$


to obtain after some computations

$$
\begin{aligned}
\zeta_{Q}(k)=2 y^{k} \zeta(2 k)+2^{3-2 k} y^{1-k} \pi \zeta(2 k-1)\left(\begin{array}{c}
2 k-2 \\
k-1
\end{array}\right) \\
\quad+\sum_{r=0}^{k-1} \sum_{n=1}^{\infty}\left(\begin{array}{c}
k-1+r \\
k-1
\end{array}\right) \frac{\sigma_{2 k-1}(n) 2^{1-2 r} \pi^{k-r}}{n^{k+r} y^{r}(k-1-r) !}[e(n \tau)+e(-n \bar{\tau})] .
\end{aligned}
$$

In deriving the above formula we used the fact that $\sigma_{1-2 k}(n)=n^{1-2 k} \sigma_{2 k-1}(n)$. Now we let $p=k-1-r$ in (2.4) and use the relation

$$
\sum_{m=1}^{\infty} \sum_{n=1}^{\infty} n^{p} m^{p+1-2 k} e(m n \tau)=\sum_{q=1}^{\infty} q^{1+p-2 k} \sigma_{2 k-1}(q) e(q \tau)
$$

to obtain the formula

$$
\begin{aligned}
\zeta_{Q}(k)=2 y^{k} \zeta(2 k)+2^{3-2 k} y^{1-k} \pi \zeta(2 k-1)\left(\begin{array}{c}
2 k-2 \\
k-1
\end{array}\right) \\
\quad+\sum_{p=0}^{k-1}\left\{\left(\begin{array}{c}
2 k-2-p \\
k-1
\end{array}\right) \frac{2^{3-2 k+2 p} \pi^{p+1}}{y^{k-1-p} p !} \sum_{m=1}^{\infty} \sum_{n=1}^{\infty} m^{p+1-2 k} n^{p}[e(m n \tau)+e(-m n \bar{\tau})]\right\} .
\end{aligned}
$$

From this equation we can obtain a partial fraction expansion for $\zeta_{Q}(k)$ as a function of $\tau$. We start with the well known relation

$$
-\pi i\left(1+2 \sum_{n=1}^{\infty} e^{2 \pi i n \tau}\right)=\pi \cot \pi \tau=\lim _{N \rightarrow \infty} \sum_{n=-N}^{N} \frac{1}{\tau+n} .
$$

We make the convention that, hereafter, all conditionally convergent doubly infinite series will be understood as limits as in (2.6). Differentiating (2.6) $p$ times, we obtain

$$
\sum_{n=1}^{\infty} n^{p} e(n \tau)=\frac{(-1)^{p+1} p !}{(2 \pi i)^{p+1}} \sum_{n=-\infty}^{\infty} \frac{1}{(\tau+n)^{p+1}} .
$$

Using these two results in (2.5) we obtain

$$
\begin{aligned}
\zeta_{Q}(k)=2 y^{k} \zeta(2 k)+2^{3-2 k} y^{1-k}\left(\begin{array}{c}
2 k-2 \\
k-1
\end{array}\right) \zeta(2 k-1) \\
+\left(\begin{array}{c}
2 k-2 \\
k-1
\end{array}\right) \frac{2^{3-2 k} \pi}{y^{k-1}} \sum_{m=1}^{\infty} m^{1-2 k}\left\{\frac{-1}{2 \pi i} \sum_{n=-\infty}^{\infty}\left[\frac{1}{m \tau+n}+\frac{1}{-m \bar{\tau}+n}\right]-1\right\} \\
+\sum_{p=1}^{k-1}\left(\begin{array}{c}
2 k-2-p \\
k-1
\end{array}\right) \frac{2^{2-2 k+p} i^{p+1}}{y^{k-1-p}} \sum_{m=1}^{\infty} \sum_{n=-\infty}^{\infty} m^{p+1-2 k}\left\{\frac{1}{(m \tau+n)^{p+1}}+\frac{1}{(-m \bar{\tau}+n)^{p+1}}\right\} .
\end{aligned}
$$

If we introduce the notation

$$
\psi_{2 k}(\tau)=\sum_{m=-\infty}^{\infty} \sum_{n=-\infty}^{\infty} \frac{1}{m^{2 k-1}(m \tau+n)},
$$


where the prime denotes that the term with $m=0$ is omitted, then we can write the above formula in the form

$$
\zeta_{Q}(k)=2 y^{k} \zeta(2 k)+(4 y)^{-k} \sum_{p=0}^{k-1}\left(\begin{array}{c}
2 k-2-p \\
k-1
\end{array}\right) \frac{(2 i y)^{p+1}(-1)^{p}}{p !}\left\{\psi_{2 k}^{(p)}(\tau)+\psi_{2 k}^{(p)}(-\bar{\tau})\right\} .
$$

We introduce the following function related to $\psi_{2 k}(\tau)$ so as to determine more easily its transformation properties under substitutions of the modular group. Define

$$
\varphi_{2 k}(\tau)=\sum_{m=-\infty}^{\infty} \sum_{n=-\infty}^{\infty} \frac{1}{m^{2 k-1}(m \tau+n)},
$$

where the prime on the summation sign indicates that $m(n)=0$ is omitted, so that

$$
\psi_{2 k}(\tau)=\varphi_{2 k}(\tau)+2 \zeta(2 k) / \tau
$$

We are now in a position to state

THEOREM 1. Let $Q(u, v)=a u^{2}+2 b u v+c v^{2}$ be a positive definite quadratic form of determinant 1 , and let $\tau=x+i y$ be associated with $Q$ as in (1.5). Then, for $k$ an integer $\geqq 2$,

$$
\begin{aligned}
\zeta_{Q}(k)=2 \zeta(2 k) y^{k}\left[1+|\tau|^{-2 k}\right] \\
+(4 y)^{-k} \sum_{p=0}^{k-1}\left(\begin{array}{c}
2 k-2-p \\
k-1
\end{array}\right)(2 y)^{p+1}\left[\varphi_{2 k}^{(p)}(\tau)+\varphi_{2 k}^{(p)}(-\bar{\tau})\right] / i^{p-1} p !
\end{aligned}
$$

Proof. If we consider separately in (2.8) the terms corresponding to $n=0$ in the doubly infinite sums, then we obtain, in view of the definition (2.11),

$$
\begin{aligned}
\zeta_{Q}(k)=2 y^{k} \zeta(2 k)+(4 y)^{-k} \sum_{p=0}^{k-1}\left(\begin{array}{c}
2 k-2-p \\
k-1
\end{array}\right)(2 y)^{p+1}\left[\varphi_{2 k}^{(p)}(\tau)+\varphi_{2 k}^{(p)}(-\bar{\tau})\right] / i^{p-1} p ! \\
+\sum_{p=0}^{k-1}\left(\begin{array}{c}
2 k-2-p \\
k-1
\end{array}\right) \frac{2^{2-2 k+p} i^{p+1}}{y^{k-1-p}} \zeta(2 k)\left[\tau^{-p-1}+(-\bar{\tau})^{-p-1}\right] .
\end{aligned}
$$

The proof will be complete upon showing that this last term is equal to $2 y^{k} \zeta(2 k) /|\tau|^{2 k}$. This is equivalent to showing that

$$
\sum_{p=0}^{k-1}\left(\begin{array}{c}
2 k-2-p \\
k-1
\end{array}\right)(2 i y)^{p+1}\left[\tau^{-p-1}+(-\bar{\tau})^{-p-1}\right]=(2 y)^{2 k} /|\tau|^{2 k}
$$

This relation can be proved by induction on $k$. Let

$$
S_{k}=\sum_{p=0}^{k}\left(\begin{array}{c}
2 k-p \\
k
\end{array}\right)(2 i y / \tau)^{p+1}
$$

For $0 \leqq p \leqq k-1$, substitute $\left(\begin{array}{c}2 k-p-1 \\ k-1\end{array}\right)+\left(\begin{array}{c}2 k-p-1 \\ k\end{array}\right)$ for $\left(\begin{array}{c}2 k-p \\ k\end{array}\right)$ in the definition of $S_{k}$. After some calculation one can show that

$$
S_{k}=(2 i y / \tau) S_{k-1}+(\tau / 2 i y) S_{k}-(1-2 i y / \tau)\left(\begin{array}{c}
2 k-1 \\
k-1
\end{array}\right)-\left(\begin{array}{c}
2 k-1 \\
k
\end{array}\right)
$$


Setting $2 i y / \tau=\xi+i \eta$, one finds that $S_{k}=\left(4 y^{2} /|\tau|^{2}\right) S_{k-1}+i \eta\left(\begin{array}{c}2 k-1 \\ k-1\end{array}\right)$ and hence

$$
S_{k}+\bar{S}_{k}=\left(4 y^{2} /|\tau|^{2}\right)\left(S_{k-1}+\bar{S}_{k-1}\right) \text {. }
$$

By calculation, $S_{1}+\bar{S}_{1}=(2 y)^{4} /|\tau|^{4}$. This proves the required relation.

Note that upon differentiating $\psi_{2 k}(\tau) 2 k-1$ times we obtain a constant multiple of the Eisenstein series (1.8). Thus $\varphi_{2 k}(\tau)$ is closely related, by (2.12), to an indefinite integral of an Eisenstein series.

3. Transformation formula of $\varphi_{2 k}(\tau)$. In this section we obtain the transformation properties of the functions $\varphi_{2 k}(\tau)$. However, for the purpose of applying these results to the summation of certain double series in the next section, we make the following more general definition.

Let $N$ be a positive integer and $a_{1}$ and $a_{2}$ integers, and define

$$
\varphi_{2 k}\left(\tau ; a_{1}, a_{2}, N\right)=\sum_{m \equiv a_{1}(N)}^{\prime} \sum_{n \equiv a_{2}(N)}^{\prime} \frac{1}{m^{2 k-1}(m \tau+n)} .
$$

In the above double sums the prime indicates that the term with $m=0$ (or $n=0$ ) is to be omitted if it could occur. As before, we understand conditionally convergent double series in the sense $\lim _{M \rightarrow \infty} \sum_{m=-M}^{M}$. First of all, we show that the above series converges uniformly on compact subsets of the upper half plane $\mathscr{H}$. Write

$$
\begin{aligned}
\sum_{n \equiv a_{2}(N)}(m \tau+n)^{-1} & =\lim _{M \rightarrow \infty} \sum_{t=-M}^{M}\left(m \tau+a_{2}+t N\right) \\
& =\lim _{M \rightarrow \infty} \sum_{1=1}^{M} \frac{\left(2 m \tau+a_{2}\right)}{\left(m \tau+a_{2}+t N\right)\left(m \tau+a_{2}-t N\right)}+\frac{\delta\left(0, a_{2}, N\right)}{\left(m \tau+a_{2}\right)},
\end{aligned}
$$

where, for integers $a, b$,

$$
\delta(a, b, N)=\left\{\begin{array}{lll}
1 & \text { if } & a \equiv b(\bmod N), \\
0 & \text { if } & a \neq b(\bmod N) .
\end{array}\right.
$$

Now we use the estimate $|c \tau+d| \geqq|d| \sin \arg \tau$, valid for $c, d$ real and $\tau \in \mathscr{H}$. For $\tau$ in a compact subset $K$ of $\mathscr{H}$ we have

$$
\left|\frac{2\left(m \tau+a_{2}\right)}{\left(m \tau+a_{2}+t N\right)\left(m \tau+a_{2}-t N\right)}\right| \leqq \frac{2\left|m \tau+a_{2}\right|}{\left|a_{2}+t N\right|\left|a_{2}-t N\right| \sin ^{2} \arg \tau}=O\left(\frac{m}{t^{2}}\right)
$$

uniformly in $K$. Thus we see that the series of (3.1) converges uniformly in $K$.

It is convenient to introduce the following notation. Let $N$ be a positive integer, $a$ an integer and let

$$
\begin{gathered}
\zeta(s ; a, N)=\sum_{n \equiv a(N)}^{\prime} n^{-s} \quad(s=\sigma+i t, \sigma \geqq 1) \\
\left(n^{-s}=e^{-s \log n}=|n|^{-s} e^{-s \arg n}, \arg n=0,-\pi\right) .
\end{gathered}
$$


Now we obtain the transformation formula

$$
\begin{gathered}
\varphi_{2 k}\left(\tau+1 ; a_{1}, a_{2}, N\right)=\varphi_{2 k}\left(\tau, a_{1}, a_{1}+a_{2}, N\right)+\zeta\left(2 k ; a_{1}, N\right)\left\{\delta\left(0, a_{1}+a_{2}, N\right) / \tau\right. \\
\left.-\delta\left(a_{1}, a_{1}+a_{2}, N\right) /(\tau+1)\right\} .
\end{gathered}
$$

We start with

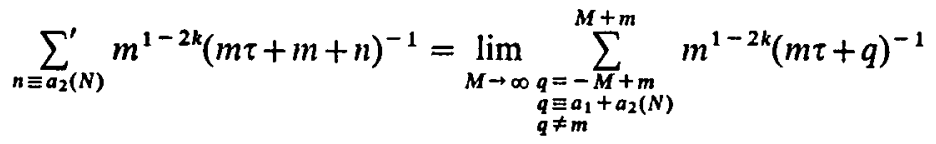

$$
\begin{aligned}
& =\lim _{M \rightarrow \infty}\left\{\sum_{\substack{q=-M-m \\
q \equiv a_{1}+a_{2}(N)}}^{M+m} m^{1-2 k}(m \tau+q)^{-1}-\frac{\delta\left(m, a_{1}+a_{2}, N\right)}{m^{2 k-1}(m \tau+m)}\right. \\
& \left.+\frac{\delta\left(0, a_{1}+a_{2}, N\right)}{m^{2 k} \tau}-\sum_{\substack{q=-M-m \\
q \equiv a_{1}+a_{2}(N)}}^{-M+m-1} m^{1-2 k}(m \tau+q)\right\} \\
& =\sum_{n \equiv a_{1}+a_{2}(N)}^{\prime} m^{1-2 k}(m \tau+n)^{-1}+\frac{\delta\left(0, a_{1}+a_{2}, N\right)}{m^{2 k} \tau}-\frac{\delta\left(m, a_{1}+a_{2}, N\right)}{m^{2 k}(\tau+1)} .
\end{aligned}
$$

The result (3.2) follows upon summing the above equation over values of $m$ for which $m \equiv a_{1}(N)$.

Now we seek the transformation formula when $\tau$ is replaced by $-1 / \tau$. We obtain this relation with the aid of the following identity:

$$
m^{1-2 k} /(m \tau+n)=\sum_{j=1}^{2 k-1}(-1)^{j+1} m^{-j} n^{j-2 k} \tau^{2 k-j-1}-n^{1-2 k} \tau^{2 k-1} /(m \tau+n),
$$

obtained by writing the left hand side in its partial fraction decomposition as a function of $m$. Then, summing (3.4) first over $n \equiv a_{2}(N)$ and then over $m \equiv a_{1}(N)$, we find that

Now consider

$$
\begin{aligned}
\varphi_{2 k}\left(\tau ; a_{1}, a_{2}, N\right)= & \sum_{j=1}^{2 k-1}(-1)^{j+1} \tau^{2 k-j-1} \zeta\left(j ; a_{1}, N\right) \zeta\left(2 k-j ; a_{2}, N\right) \\
& -\tau^{2 k-1} \sum_{m \equiv a_{1}(N)}^{\prime} \sum_{n \equiv a_{2}(N)}^{\prime} n^{1-2 k}(m \tau+n)^{-1} .
\end{aligned}
$$

$$
\begin{aligned}
\varphi_{2 k}\left(-1 / \tau ; b_{1}, b_{2}, N\right) & =\tau \sum_{m \equiv b_{1}}^{\prime} \sum_{n \equiv b_{2}}^{\prime} m^{1-2 k}(-m+n \tau)^{-1} \\
& =\tau \sum_{s=-\infty}^{\infty} \sum_{t=-\infty}^{\prime}\left(b_{1}+s N\right)^{1-2 k}\left[-b_{1}-s N+\left(b_{2}+\tau N\right) \tau\right]^{-1} \\
& =\tau \sum_{s=-\infty}^{\infty} \sum_{t=-\infty}^{\infty}\left(b_{1}-s N\right)^{1-2 k}\left[-b_{1}+s N+\left(b_{2}+t N\right) \tau\right]^{-1} \\
& =-\tau \sum_{s=-\infty}^{\infty} \sum_{t=-\infty}^{\infty}\left(-b_{1}+s N\right)^{1-2 k}\left[-b_{1}+s N+\left(b_{2}+t N\right) \tau\right]^{-1} \\
& =-\tau \sum_{n \equiv b_{2}} \sum_{m=-b_{1}} m^{1-2 k}(n \tau+m)^{-1} .
\end{aligned}
$$


The interchange of order in this last step is seen to be valid by summing the terms corresponding to $\pm t$ together. The resulting sum can be shown to be absolutely convergent in $\mathscr{H}$ by using the estimate $|c \tau+d| \geqq|c| y$, valid for $\tau \in \mathscr{H}$ and $c$ and $d$ real. One finds that the terms of the sum are $O\left(s^{2-2 k} t^{2}\right)$, where the constant depends upon $b_{1}, b_{2}$ and $\tau$. It follows that (3.5) can be written in the form

$$
\begin{aligned}
\varphi_{2 k}\left(\tau ; a_{1}, a_{2}, N\right)= & \sum_{j=1}^{2 k-1}(-1)^{j+1} \tau^{2 k-j-1} \zeta\left(j ; a_{1}, N\right) \zeta\left(2 k-j ; a_{2}, N\right) \\
& +\tau^{2 k-2} \varphi_{2 k}\left(-1 / \tau ;-a_{2}, a_{1}, N\right) .
\end{aligned}
$$

This becomes, upon replacing $a_{1}, a_{2}$ by $a_{2},-a_{1}$,

$$
\begin{aligned}
& \tau^{2 k-2} \varphi_{2 k}\left(-1 / \tau ; a_{1}, a_{2}, N\right) \\
& \quad=\varphi_{2 k}\left(\tau ; a_{2},-a_{1}, N\right)+\sum_{j=1}^{2 k-1}(-1)^{j} \tau^{2 k-j-1} \zeta\left(j ; a_{2}, N\right) \zeta\left(2 k-j ;-a_{1}, N\right) .
\end{aligned}
$$

Grosswald [4] derived a functional equation of this type for

$$
F(\tau)=-\varphi_{2 k}(\tau) / 4 \pi i-\zeta(2 k) / 2 \pi i \tau-\zeta(2 k-1) / 2,
$$

which corresponds to the case $N=1, a_{1}=a_{2}=0$. Now we are in position to state

THEOREM 2. Let $V=\left(\begin{array}{ll}a & b \\ c & d\end{array}\right) \in \Gamma(1)$. Then the functions $\varphi_{2 k}(\tau)$ defined in (3.1) satisfy the transformation formula

$$
(c \tau+d)^{2 k-2} \varphi_{2 k}\left(V \tau ; a_{1}, a_{2}, N\right)=\varphi_{2 k}\left(\tau ; a a_{1}+c a_{2}, b a_{1}+d a_{2}, N\right)+r(\tau, V),
$$

where $r(\tau, V)$ is a rational function of $\tau$ which depends upon $a_{1}, a_{2}$ and $V$. The transformation formulas (3.3) and (3.7) give $r(\tau, V)$ for the generators $V=U$ and $V=T$, respectively, of $\Gamma(1)$.

Proof. The elements of $\Gamma(1)$ can be written as words in $U=\left(\begin{array}{ll}1 & 1 \\ 0 & 1\end{array}\right)$ and $T=\left(\begin{array}{cc}0 & 1 \\ -1 & 0\end{array}\right)$. The proof is by induction on the word length. The result is clearly true when $V= \pm I$. Suppose that it is true for all elements of $V$ that can be written as a word in $U$ and $T$ of length less than $n$. Consider $M \in \Gamma(1)$, which can be written in the form $V T, V T^{-1}, V U$, $V U^{-1}$, where $V$ is a word in $U$ and $T$ of length less than $n$. We replace $\tau$ by $T \tau, T^{-1} \tau, U \tau$ or $U^{-1} \tau$, as the case may be, in (3.8); for example, on replacing $\tau$ by $T \tau$, we see with $V=\left(\begin{array}{ll}a & b \\ c & d\end{array}\right)$ that

$$
(c T \tau+d)^{2 k-2} \varphi_{2 k}\left(V T \tau ; a_{1}, a_{2}, N\right)=\varphi_{2 k}\left(T \tau ; a a_{1}+c a_{2}, b a_{1}+d a_{2}, N\right)+r(T \tau, V)
$$

or

$$
(d \tau-c)^{2 k-2} \varphi_{2 k}\left(V T \tau ; a_{1}, a_{2}, N\right)=\tau^{2 k-2} \varphi_{2 k}\left(T \tau ; a a_{1}+c a_{2}, b a_{1}+d a_{2}, N\right)+\tau^{2 k-2} V(T \tau, V) .
$$

By (3.7),

$$
(d \tau-c)^{2 k-2} \varphi_{2 k}\left(V T \tau ; a_{1}, a_{2}, N\right)=\varphi_{2 k}\left(\tau ; b a_{1}+d a_{2},-a a_{1}-c a_{2}, N\right)+r(\tau, T)+\tau^{2 k-2} r(T \tau, V) .
$$


Now note that $V T=\left(\begin{array}{ll}b & -a \\ d & -c\end{array}\right)$. This completes the proof in this case. The other three cases are similar; in fact, since $T^{-1}=-T$, this case need not be considered. We remark that the rational functions $r(\tau, V)$ satisfy the relation

$$
r\left(V_{1} V_{2}, \tau\right)=r_{2 k}\left(V_{2}, \tau\right)+\left(c_{2} \tau+d_{2}\right)^{2 k-2} r_{2 k}\left(V_{1}, V_{2} \tau\right)
$$

where

$$
V_{i}=\left(\begin{array}{ll}
a_{i} & b_{i} \\
c_{i} & d_{i}
\end{array}\right) \quad(i=1,2)
$$

4. Applications of the transformation formulas to the summation of certain series. We are able to apply the transformation formulas (3.3) and (3.7) to determine $\varphi_{2 k}\left(i ; a_{1}, a_{2}, N\right)$ and $\varphi_{2 k}\left(\rho ; a_{1}, a_{2}, N\right), \rho=\frac{1}{2}(-1+i \sqrt{ } 3)$ in some cases. This is done in part (a) of this section. I believe this to be of interest in its own right. In parts (b), (c) and (d) of this section we apply these results to the summation of some series.

(a) Calculation of $\varphi_{2 k}\left(\tau ; a_{1}, a_{2}, N\right)$.

Case 1. $a_{1}=a_{2}=0, N=1$. From $(3.7)$, if $k \equiv 0(\bmod 2)$, we find that

$$
\varphi_{2 k}(i)=2 i \sum_{j=1}^{k-1}(-1)^{j \zeta}(2 j) \zeta(2 k-2 j) \text {. }
$$

If $k \neq 1(\bmod 3)$, then, from (3.7),

$$
\rho^{2 k-2} \varphi_{2 k}(\rho+1)=\varphi_{2 k}(\rho)+4 \sum_{j=1}^{k-1} \rho^{2 k-2 j-1} \zeta(2 j) \zeta(2 k-2 j),
$$

since $-1 / \rho=\rho+1$. From (3.2), we see that

Thus

$$
\varphi_{2 k}(\rho+1)=\varphi_{2 k}(\rho)+2 \zeta(2 k) / \rho(\rho+1)
$$

$$
\left(\rho^{2 k-2}-1\right) \varphi_{2 k}(\rho)=4 \sum_{j=1}^{k-1} \rho^{2 k-2 j-1} \zeta(2 j) \zeta(2 k-2 j)-2 \zeta(2 k) \rho^{2 k-3} /(\rho+1) .
$$

Case 2. $a_{1}=a_{2}=1, N=2$. From (3.7) we obtain that, if $k \equiv 0(\bmod 2)$, then

$$
\varphi_{2 k}(i ; 1,1,2)=\frac{i}{2} \sum_{j=1}^{k-1}(-1)^{j \zeta}(2 j ; 1,2) \zeta(2 k-2 j ; 1,2) .
$$

Case 3. $a_{1}=1, a_{2}=2, N=3$. It follows from (3.7) that

$$
\rho^{2 k-2} \varphi_{2 k}(\rho+1 ; 1,1,3)=\varphi_{2 k}(\rho ; 1,-1,3)+\sum_{j=1}^{k-1}(-1)^{j+1} \rho^{2 k-j-1} \zeta(j ; 1,3) \zeta(2 k-j ; 2,3)
$$

and from (3.2) that

Thus

$$
\varphi_{2 k}(\rho+1 ; 1,1,3)=\varphi_{2 k}(\rho ; 1,2,3)
$$

$$
\left(\rho^{2 k-2}-1\right) \varphi_{2 k}(\rho ; 1,2,3)=\sum_{j=1}^{k-1}(-1)^{j} \rho^{2 k-j-1} \zeta(j ; 1,3) \zeta(2 k-j ; 2,3) \text {. }
$$


We now give a short table of values of the function $\varphi_{2 k}\left(\tau ; a_{1}, a_{2}, N\right)$.

$$
\begin{aligned}
\varphi_{4}(i) & =-i \pi^{4} / 18 & \varphi_{8}(i) & =-13 i \pi^{8} / 28350 \\
\varphi_{4}(i ; 1,1,2) & =-i \pi^{4} / 32 & & \varphi_{4}(\rho)=\pi^{4}(1-i 3 \sqrt{3}) / 90 \\
\varphi_{8}(i ; 1,1,2) & =-7 i \pi^{8} / 23040 & \varphi_{6}(\rho) & =(3-8 i \sqrt{3}) \pi^{6} / 2835
\end{aligned}
$$

Table 1

(b) Now we compute some series associated with the functions $\varphi_{2 k}\left(\tau ; a_{1}, a_{2}, N\right)$. Starting with definition (3.1), if we multiply numerator and denominator of the summands by $m \bar{\tau}+n$, equate real and imaginary parts and use the relation (1.5), we obtain

and

$$
\sum_{m \equiv a_{1}(N)}^{\prime} \sum_{n \equiv a_{2}(N)}^{\prime} m^{2-2 k} / Q(n, m)=-\operatorname{Im} \varphi_{2 k}\left(\tau ; a_{1}, a_{2}, N\right)
$$

$$
\sum_{m \equiv a_{1}(N)}^{\prime} \sum_{n \equiv a_{2}(N)}^{\prime} n m^{1-2 k} / Q(n, m)=\operatorname{Im} \tau \varphi_{2 k}\left(\tau ; a_{1}, a_{2}, N\right) .
$$

Another type of series can be obtained using the partial fraction expansion (2.6) for $\pi \cot \pi \tau$. We see that

$$
\frac{\pi}{N} \cot \pi \frac{m \tau+a_{2}}{N}=\frac{1}{N} \sum_{t=-\infty}^{\infty} 1 /\left[\left(m \tau+a_{2}\right) / N+t\right]
$$

and so, from (3.1),

$$
\varphi_{2 k}\left(\tau ; a_{1}, a_{2}, N\right)=(\pi / N) \sum_{m \equiv a_{1}(N)}^{\prime} \frac{\cot \pi\left(m \tau+a_{2}\right) / N}{m^{2 k-1}}-\frac{\delta\left(0, a_{2}, N\right)}{\tau} \cdot \zeta\left(2 k ; a_{1}, N\right) .
$$

(c) We can now put the results of (a) and (b) together to sum various series.

First, if $k \equiv 0(\bmod 2)$, then we have, from (4.1), (4.3) and (4.5), that

$$
\sum_{m}^{\prime} \sum_{n}^{\prime} m^{2-2 k} /\left(m^{2}+n^{2}\right)=2 \sum_{j=1}^{k-1}(-1)^{j+1} \zeta(2 j) \zeta(2 k-2 j)
$$

and

$$
\sum_{m=1(2)} \sum_{n=1(2)} m^{2-2 k} /\left(m^{2}+n^{2}\right)=\frac{1}{2} \sum_{j=1}^{k-1}(-1)^{j+1} \zeta(2 j ; 1,2) \zeta(2 k-2 j ; 1,2),
$$

where the prime denotes that the term with $m(n)=0$ is omitted from the sum. These formulas also have an elementary derivation starting from a partial fraction expansion similar to (3.4). $\zeta(2 j ; 1,2)$ can be computed from $[1$, p. 807]

$$
\sum_{k=0}^{\infty} 1 /(2 k+1)^{2 n}=\frac{1}{2}\left(2^{2 n}-1\right) \pi^{2 n}\left|B_{2 n}\right| /(2 n) !
$$

If we still require that $k \equiv 0(\bmod 2)$, then, from $(4.7),(4.5)$ and $(4.1)$, we find that

$$
\sum_{m=1}^{\infty} \operatorname{coth} \pi m / m^{2 k-1}=\frac{1}{\pi} \sum_{j=1}^{k-1}(-1)^{j+1} \zeta(2 j) \zeta(2 k-2 j)+\zeta(2 k) / \pi
$$


Similarly, from (4.7), (4.5) and (4.3), we obtain

$$
\sum_{m=0}^{\infty} \frac{\tanh \pi(2 m+1) / 2}{(2 m+1)^{2 k-1}}=\frac{1}{2 \pi} \sum_{j=1}^{k-1}(-1)^{j+1} \zeta(2 j ; 1,2) \zeta(2 k-2 j ; 1,2)
$$

The formula (4.11) has an interesting history. The case $k=4$ was one of the 120 formulas that Ramanajan sent Hardy in his celebrated letter [8]. In 1928 Watson [12] proved (4.11) by the method of contour integration. So far as I know, (4.12) is new.

We conclude this part with a short table of sums of series obtainable from the values in Table 1:

$$
\begin{gathered}
\sum^{\prime} \sum^{\prime} 1 / m^{6}\left(m^{2}+n^{2}\right)=13 \pi^{8} / 28350, \sum_{m \equiv 1(2)} \sum_{n=1(2)} 1 / m^{2}\left(m^{2}+n^{2}\right)=\pi^{4} / 32 \\
\sum_{m=1(2)} \sum_{n=1(2)} 1 / m^{6}\left(m^{2}+n^{2}\right)=7 \pi^{8} / 23040, \sum_{m}^{\prime} \sum_{n}^{\prime} 1 / m^{2}\left(m^{2}-m n+n^{2}\right)=\pi^{4} \sqrt{ } 3 / 30 \\
\sum_{m=1}^{\infty} \frac{\operatorname{coth} \pi m}{m^{3}}=7 \pi^{3} / 180, \sum_{m=0}^{\infty} \frac{\tanh \pi(2 m+1) / 2}{(2 m+1)^{3}}=\pi^{3} / 32
\end{gathered}
$$

Table 2

(d) Finally we mention the connection of these results with the zeta function of a number field. Let $K=\mathbf{Q}(\sqrt{D})$, where $D$ is square free, have discriminant $d$; then the zeta function of $K$ [6] satisfies

$$
\zeta(s, d)=\sum_{a} N a^{-s}=\zeta(s) L(s, d),
$$

where summation is over all nonzero integral ideals $a$ of $K$ and

$$
L(s, d)=\sum_{n=1}^{\infty}\left(\frac{d}{n}\right) / n^{s}
$$

In the case of $D=-1$, we have $d=-4$ and

$$
L(s,-4)=\sum_{n=0}^{\infty}(-1)^{n} /(2 n+1)^{s} .
$$

If $D=-3$, then $d=-3$ and

$$
L(s,-3)=(2 / \sqrt{3}) \sum_{n=1}^{\infty}(\sin 2 n \pi / 3) n^{s} .
$$

From the definition of the norm of an integer in $Q(\sqrt{-1})$ and $Q(\sqrt{-3})$, we see that

$$
\left.\begin{array}{l}
\zeta_{Q_{1}}(s)=4 \zeta(s,-4)=4 \zeta(s) L(s,-4), \\
\zeta_{Q_{2}}(s)=6(\sqrt{3} / 2)^{s} \zeta(s,-3)=6(\sqrt{3} / 2)^{s} \zeta(s) L(s,-3),
\end{array}\right\}
$$

where $Q_{1}=m^{2}+n^{2}, Q_{2}=(2 / \sqrt{3})\left(m^{2}-m n+n^{2}\right)$. There are known formulas [1] for $L(s,-4)$ and $L(s,-3)$ when $s$ is an odd integer. We can sum one final series in terms of $L(2,-4)$, 
which is known as Catalan's constant [1, p. 807]. From (2.13),

so

$$
\zeta_{Q_{1}}(2)=4 \zeta(4)+\left(\varphi_{4}(i) i+\varphi_{4}^{\prime}(i)\right) / 2 \text {; }
$$

$$
\begin{aligned}
4 \zeta(2) L(2,-4) & =4 \zeta(4)+\zeta^{2}(2)+\varphi_{4}^{\prime}(i) / 2, \\
\varphi_{4}^{\prime}(i) & =\left(120 \pi^{2} L(2,-4)-13 \pi^{4}\right) / 180 .
\end{aligned}
$$

From (4.7) we see that $\varphi_{4}^{\prime}(i)=2 \pi^{2} \sum 1 /\left(m^{2} \sinh m \pi\right)-2 \zeta(4)$; thus

$$
\sum_{m=1}^{\infty} 1 /\left(m^{2} \sinh m \pi\right)=\left(120 L(2,-4)-11 \pi^{2}\right) / 180 \text {. }
$$

5. Applications to the Riemann zeta function for odd arguments. In this section we rederive the results of Grosswald [4] and add a few connections with the previous section. Let

$$
F(\tau)=\varphi_{2 k}(\tau) / 4 \pi i-\zeta(2 k) / 2 \pi i \tau-\zeta(2 k-1) / 2
$$

then from (2.11) and (2.6) we see that

$$
F(\tau)=\sum_{m=1}^{\infty} m^{-(2 k-1)} \sum_{n=1}^{\infty} e^{2 \pi i n n \tau}=\sum_{m=1}^{\infty} 1 /\left[m^{2 k-1}\left(e^{-2 \pi i m r}-1\right)\right]
$$

and this is the function considered by Grosswald. Solving for $\zeta(2 k-1)$ in $(5.1)$, setting $\tau=i$ and using (4.1), we find that, if $2 k \equiv 0(\bmod 4)$, then

$$
\zeta(2 k-1)=\zeta(2 k) / \pi+(2 / \pi) \sum_{j=1}^{k-1} \zeta(2 k-2 j) \zeta(2 j)(-1)^{j+1}-2 \sum_{m=1}^{\infty} 1 /\left[m^{2 k-1}\left(e^{2 \pi m}-1\right)\right] .
$$

Now, if $2 k \equiv 0(\bmod 4)$, we differentiate (5.1) and (3.7) (with $\left.N=1, a_{1}=a_{2}=0\right)$ and set $\tau=i$ to obtain

$$
\begin{array}{r}
\zeta(2 k-1)=k \zeta(2 k) / \pi(k-1)+\sum_{j=1}^{k-1} \zeta(2 k-2 j) \zeta(2 j)(2 k-2 j-1)(-1)^{k-j} / \pi(k-1) \\
+\frac{4 \pi}{k-1} \sum_{m=1}^{\infty} 1 /\left[m^{2 k-2}\left(e^{2 \pi m}-1\right)^{2}\right]-2 \sum_{m=1}^{\infty} 1 /\left[m^{2 k-1}\left(e^{2 \pi m}-1\right)\right] .
\end{array}
$$

We note in passing that, by using (4.2), similar formulas could be obtained involving the series

$$
\sum_{m=1}^{\infty} 1 /\left[m^{2 k-1}\left((-1)^{m} e^{\pi m \sqrt{3}}-1\right)\right]
$$

We now consider the connections with $\zeta(3)$. Noting from $(2.11)$ that $\varphi_{6}(\bar{\tau})=\overline{\varphi_{6}(\tau)}$ and $\varphi_{6}(-\tau)=-\varphi_{6}(\tau)$, we obtain from (2.13) with $k=3$ the relation

$$
\zeta_{Q}(3)=2 \zeta(6) y^{3}\left(1+|\tau|^{-6}\right)-3 \operatorname{Im} \varphi_{6}(\tau) / 8 y^{2}+3 \operatorname{Re} \varphi_{6}^{\prime}(\tau) / 8 y+\operatorname{Im} \varphi_{6}^{\prime \prime}(\tau) / 8
$$

Now, specializing this result with $\tau=i, Q=Q_{1}=m^{2}+n^{2}$, we find on comparing (4.17) that

$$
4 \zeta(3) L(3,-4)=4 \zeta(6)+3 i \varphi_{6}(i) / 8+3 \varphi_{6}^{\prime}(i) / 8-i \varphi_{6}^{\prime \prime}(i) / 8 \text {. }
$$

After differentiating (3.7) and setting $\tau=i$, we get the relation $2 i \varphi_{6}(i)+\varphi_{6}^{\prime}(i)=4 \zeta(2) \zeta(4)$. Using the known value $L(3,-4)=\pi^{3} / 32[1$, p. 808], we have

$$
\zeta(3)=\frac{53}{945} \pi^{3}-\frac{1}{\pi^{3}}\left(3 i \varphi_{6}(i)+i \varphi_{6}^{\prime \prime}(i)\right)
$$


Now in a similar way we consider the case $\tau=\rho$ corresponding to $Q=Q_{2}=$ $(2 / \sqrt{3})\left(m^{2}-m n+n^{2}\right)$. First we compute $\varphi_{6}^{\prime}(\rho)$. Using the relations $-1 / \rho=\rho+1,(4.10)$, (1.10a), (1.10b) and the formulas obtained from them by differentiation with respect to $\tau$, we find after some calculations that $\varphi_{6}^{\prime}(\rho)=\left(\pi^{6} / 3^{4} \cdot 5 \cdot 7\right)(-4+3 i \sqrt{3})$. Next using the relation [1, p. 809] $\sum_{1}^{\infty}(\sin k \pi x) / k^{3}=\left(x^{3}-3 x^{2} / 2+x / 2\right) \pi^{3} / 6$ with $x=\frac{1}{3}$ and $(4.18)$, we see that $L(3,-3)=$ $4 \pi^{3} \sqrt{3} / 3^{5}$. Thus from (4.17) we see that

$$
\zeta_{Q_{2}}(3)=\left(\pi^{3} / 9\right) \zeta(3)
$$

Now substituting the values for $\varphi_{6}(\rho)$ and $\varphi_{6}^{\prime}(\rho)$ into (5.5), we derive

$$
\zeta(3)=\pi^{3} \sqrt{3} / 42+9 \operatorname{Im} \varphi_{6}^{\prime \prime}(\rho) / 8 .
$$

It is seen that, if one could compute $\varphi_{6}(\tau), \varphi_{6}^{\prime}(\tau)$ and $\varphi_{6}^{\prime \prime}(\tau)$ for $\tau=i$ or $\tau=\rho$, we would then have computed $\zeta(3)$. These computations seem very difficult.

Added in proof. A. Hurwitz employed devices similar to (3.4) in order to obtain transformation formulas of modular forms (Cf. Werke I (Basel, 1962), 23ff. and 583ff.). Joseph Lewittes, in his paper "Analytic continuation of the series $\sum(m+n z)^{-s}$ ", Trans. Amer. Math. Soc. 159 (1971), 505-509, essentially established the transformation formula (1.10b) when $k \leqq 0$, by different methods. Grosswald has generalized the results of [4] in his paper

" Remarks concerning the values of the Riemann zeta function at integral odd arguments", J. Number Theory 4 (1972), 225-235.

\section{REFERENCES}

1. M. Abromowitz and I. A. Stegun, editors, Handbook of mathematical functions, National Bureau of Standards, Applied Mathematics Series 55 (Washington, 1964).

2. P. T. Bateman and E. Grosswald, On Epstein's zeta function, Acta Arith. 9 (1964), 365-373. 86-110.

3. S. Chowla and A. Selberg, On Epstein's zeta function, J. Reine Angew. Math. 227 (1967),

4. E. Grosswald, Die Werte der Riemannschen Zetafunktion an ungeraden Argumentstellen, Nachr. Akad. Wiss. Göttingen Math.-Phys. Kl. IIa (1970, Nr. 2), 9-13.

5. R. C. Gunning, Lectures on modular forms, Ann. of Math. Studies 48 (Princeton, 1962).

6. E. Hecke, Algebraische Zahlen (New York, 1948).

7. J. Lehner, Discontinuous groups and automorphic functions, Amer. Math. Soc. Math. Surveys No. 8 (Providence, R.I., 1964).

8. S. Ramanujan, Collected papers (Cambridge, 1927).

9. R. A. Rankin, A minimum problem for the Epstein zeta function, Proc. Glasgow Math. Assoc. 1 (1953), 149-158.

10. G. Sansone and J. Gerretson, Lectures on the theory of functions of a complex variable I (Groningen, 1960).

11. C. L. Siegel, Lectures on advanced analytic number theory (Tata Institute, Bombay, 1961).

12. G. N. Watson, Theorems stated by Ramanujan II, J. London Math. Soc. 3 (1928), 216-225.

13. G. N. Watson, Theory of Bessel functions (Cambridge, 1966).

UNIVERSITY OF WISCONSIN

MADISON, WisCONSIN 